\title{
Higher order curvature information and its application in a modified diagonal Secant method
}

\begin{abstract}
ABSTRCT
A secant equation (quasi-Newton) has one of the most important rule to find an optimal solution in nonlinear optimization. Curvature information must satisfy the usual secant equation to ensure positive definiteness of the Hessian approximation. In this work, we present a new diagonal updating to improve the Hessian approximation with a modifying weak secant equation for the diagonal quasi-Newton (DQN) method. The gradient and function evaluation are utilized to obtain a new weak secant equation and achieve a higher order accuracy in curvature information in the proposed method. Modified DQN methods based on the modified weak secant equation are globally convergent. Extended numerical results indicate the advantages of modified DQN methods over the usual ones and some classical conjugate gradient methods.
\end{abstract}

Keyword: Nonlinear optimization; Secant equation; Curvature information; Hessian approximation; Modified diagonal quasi-Newton method 